\title{
Approximate Local Poincaré Spacetime Symmetry in General Relativity
}

Samuel C. Fletcher

\begin{abstract}
How does general relativity reduce, or explain the success of, special relativity? Answering this question, which Einstein took as a desideratum in the formulation of the former, is of acknowledged importance, yet there continues to be disagreement about how exactly it is best answered. I advocate here that part of the best answer involves showing that every relativistic spacetime has an approximate local Poincaré spacetime symmetry group, the spacetime symmetry group of Minkowski spacetime. This explains the application of Minkowski spacetime concepts that depend on, e.g., the conserved quantities that spacetime symmetries guarantee. I contrast this approach with another that instead invokes the strong equivalence principle, which focuses on the distinct notion of Poincaré invariance of dynamical equations. After showing with some examples that neither notion is necessary for the other, I use those examples to illuminate contrasting positions on the explanatory role of local approximate spacetime geometry, defending Torretti (1996) against criticisms by Brown and Pooley (2001). Finally, I acknowledge that establishing approximate local Poincaré spacetime symmetry is not a complete answer to the explanatory question with which I led, discussing in the concluding section further work that could lead to an answer. This includes specifying the circumstances under which matter fields in a general relativistic spacetime "behave" locally like those in Minkowski spacetime.
\end{abstract}

S. C. Fletcher

University of Minnesota, Twin Cities, Minneapolis, USA, e-mail: scfletch@umn.edu 


\section{Introduction: Explanation, Reduction, and Symmetry}

\subsection{Explanation and Reduction}

Several constraints and heuristics guided Einstein's endeavor to find an acceptable relativistic theory of gravitation. One was such a theory's relationship to the special theory of relativity (SR). Not only should it reduce to SR when substantial gravitational fields were absent (Renn and Sauer, 1998, p. 97), but also the interpretation of its metric components should mirror that for SR (Norton, 1984, p. 261), and it should reproduce the kinematics of relativistic continua (Janssen, 2014, p. 211). In a word - though, perhaps, not one Einstein would have used - a relativistic theory of gravitation had to explain the success of SR. Despite this potential anachronism, it captures succinctly the demand incumbent on a relativistic theory of gravitation: to answer how applications of SR were as successful as they indeed were, even though they idealized away all gravitational effects acknowledged as ever present.

There is no debate about whether the culmination of Einstein's endeavor in the general theory of relativity (GR) fulfills this demand. It does, but how it best does it not often agreed upon. My goal in this note is to exhibit in some novel detail one aspect of my preferred explanation, which is to delineate the circumstances under which successfully applied (partial) solutions, or models, of fields in SR approximate those in GR that are in those circumstances at least as successful. In this sense, GR reduces to SR. It is not a reduction in the usual philosophers' sense of deducing SR from GR, for the two are generally incompatible when applied to the self-same phenomena. Rather, it is somewhat more in the vein of "physicists' sense of reduction" as described by Nickles (1973) and elaborated by Ehlers (1986): it shows how the (typically) newer, more expansive theory represents more phenomena successfully, and accounts for how the (typically) older, less expansive theory represented phenomena successfully by showing that it well approximates the former in these cases.

All that said, this is not the occasion for a general disquisition on the concepts of reduction or explanation. I shall not defend the claims that accounting for the success of SR within GR is a form of both, but I also shall not draw from accounts of explanation or reduction in my arguments. So for those skeptical of these claims, my use of these terms may serve as a label for the elaboration of how the domain of application of SR is subsumed through approximation and correction to that of GR. ${ }^{1}$

\footnotetext{
${ }^{1}$ Rosaler (2019) declines from calling this sort of reduction-as-domain-subsumption an explanation, citing the deep controversies over accounts of explanation. I agree with him that the present sense of explanation is not well modeled, e.g., within the deductive-nomological or other standard philosophical accounts of scientific explanation, but so much the worse for those accounts.
} 


\subsection{Spacetime Symmetries}

Another reason I do not focus on defending these claims is that I only treat presently a fragment of the whole project of reducing or explaining the success of SR. In particular, I focus on the successful application of spacetime symmetries. Given any relativistic spacetime- that is, a model $(M, g)$, with $M$ a four-dimensional smooth manifold and $g$ a smooth Lorentz signature metric on it $^{2}$ - consider the collection of diffeomorphisms $\psi: M \rightarrow M$ such that the pushforward of the spacetime metric is itself, i.e., $\psi_{*}(g)=g$. In a word, a spacetime symmetry is an automorphism of a relativistic spacetime; it maps the spacetime metric back onto itself within the same spacetime. ${ }^{3}$ The collection of all such maps forms a group under composition, hence constitutes the spacetime symmetry group of $(M, g)$. There are many fewer spacetimes with symmetries than those without, so their presence greatly restricts the range of possible states of affairs and spacetime structures.

When a one-parameter family of spacetime symmetries is generated infinitesimally from a smooth vector field $\kappa$ on $M$, it also gives rise to conserved quantities. ${ }^{4}$ In more detail, suppose that $\kappa$ Lie-derives the metric $g$, i.e., $£_{\kappa} g=0$, in which case $\kappa$ is known as a Killing vector field. ${ }^{5}$ Killing vector fields can give rise to two sorts of conserved quantities, one associated with the worldlines of free particles, and another associated with divergence-free, localized symmetric tensor fields, like those representing energy-momentum (Malament, 2007, §2.7).

Consider first any particle with worldline $\gamma: I \rightarrow M$, where $I \subseteq \mathbb{R}$, and tangent vector field $\xi$ on $\gamma[I]$. If $\gamma$ is a geodesic and $\kappa$ is a Killing vector field, then $J=\xi^{a} \kappa_{a}$ is constant on $\gamma[I]$. These conserved quantities can often be interpreted as energy, linear and angular momentum, and so on. Consider second any symmetric tensor field $T^{a b}$ that is divergence-free, i.e., $\nabla_{a} T^{a b}=0$. Suppose also that it is localized, in the sense that it vanishes outside some timelike world tube (Malament, 2007, p. 255). Then one can integrate $T^{a b} \kappa_{a}$ over any spacelike slice of the tube, such that the resulting quantity is constant across slices and independent of slicing. These quantities can often be interpreted analogously to those for point particles. This pro-

${ }^{2}$ I also assume that $M$ is connected, Hausdorff, and paracompact, and that the metric signature is $(+---)$. Throughout, roman sub- and superscripts denote abstract indices, while Greek and numerical ones denote components in a contextually specified basis. (See, e.g., Wald (1984, §2.4) for more on abstract index notation.) When an expression does not involve index contraction, I will often omit the indices to reduce notational clutter when no confusion should arise from doing so.

${ }^{3}$ I am implicitly using the identity map on $M$ to compare the image of the pushforward with its argument. This is ultimately a convention: there is nothing mathematically or representationally privileged about the identity over any other diffeomorphism of the manifold, but choosing a different standard of comparison would yield an entirely representationally equivalent set of spacetime symmetries (Fletcher, 2018b). This is all because diffeomorphisms are the isomorphisms in the category of smooth manifolds.

${ }^{4}$ Not all spacetime symmetries are such: consider so-called "discrete" symmetries such as reflections or time-reversal.

5 This condition is equivalent with $\kappa$ satisfying Killing's equation, $\nabla_{(a} \kappa_{b)}=0$, where $\nabla$ is the Levi-Civita covariant derivative operator compatible with the metric $g$. The Killing vector fields also form a Lie algebra, which will play a role in section 3 . 
vides a sense in which localized but extended bodies also have conserved quantities as determined by these spacetime symmetries.

A general relativistic spacetime may not have any non-trivial such spacetime symmetries (i.e., its spacetime symmetry group may consist only of the identity). By contrast, Minkowski spacetime has ten linearly independent Killing vector fields, the maximal number that a spacetime can have (Hall, 2004, Theorem 10.2.iii). These fields generate the symmetry group known as the Poincaré group (or, less elegantly, the inhomogeneous Lorentz group). It consists of four one-parameter families of symmetries corresponding to (spatial and temporal) translations, three corresponding to spatial rotations about a fixed axis, and three corresponding to boosts, which can be understood as linear transformations of Minkowski spacetime considered as an affine space. These symmetries are important in SR because their presence corresponds with both a restriction on which types of matter dynamics are possible and the presence of conserved quantities. The standard coordinates of Minkowski spacetime in SR are well-adapted to these symmetries: the congruences of the coordinate fields are integral curves of the spacetime symmetries, and the metric components expressed in their values are independent of them (Hall, 2004, p. 294-5).

Clearly many successful applications of SR appeal to these symmetries and conserved quantities, yet when de-idealized and treated within GR, these symmetries disappear. How does one thus account for the successful but idealized application of special relativity in these circumstances? I draw on a novel account of approximate spacetime symmetries (Fletcher, 2018a) to show, in section 3.1, that every relativistic spacetime has approximate local Poincaré symmetry. Although a generic spacetime of GR has no non-trivial spacetime symmetries, there is nevertheless a sense in which every spacetime approximately has maximal spacetime symmetry, if only locally. Readers thoroughly familiar with relativity theory ought not be surprised by this conclusion, but what is novel in my account is the specific details of what this means and how it comes about.

\subsection{Outline of the Remainder}

To motivate my account, I first consider another approach to understanding the "local validity" of SR in section 2, one based on the so-called strong equivalence principle. Such approaches are venerable in the foundational literature on spacetime theories, so one might wonder why the new formal apparatus I seek to introduce is really necessary. I show that while, superficially, this approach via the strong equivalence principle is concerned with the same question, on closer examination is it concerned with one that is slightly different, about Poincare invariance-namely, what sorts of coordinate transformation preserve the form of certain equations. After presenting in section 3.1 how one can account in part for the success of SR in terms of approximate local spacetime symmetries, I describe in section 3.2 how Poincaré invariance of equations is neither necessary nor sufficient to account for the suc- 
cessful application of Poincaré symmetry in local regions of a generic relativistic spacetime.

Due to the subtly different explanandum to which the strong equivalence principle is typically applied, perhaps this should not come as a great surprise. But, in the slightly digressive next section (section 4), I show how appreciating these examples sheds light on an old debate between Torretti (1996) and Brown and Pooley (2001) about what local approximate geometry does and doesn't explain. Torretti claims that the local approximate Minkowski geometry of any relativistic spacetime entails the local Lorentz invariance of dynamical laws "referred to local Lorentz charts," while Brown denies this. This new light vindicates Torretti, properly interpreted, from the charge of a non sequitur.

Although the application of Poincaré symmetry in local regions of a generic relativistic spacetime accounts in part for the success of SR, it does not account for it in total. Thus, in the concluding section 5 , after summarizing the preceding sections, I discuss some of the limitations of focusing on spacetime symmetries only. In particular, it does not necessarily account for the well-approximation of the values of observable matter fields in a generic GR spacetime by those of corresponding fields in (a region of) Minkowski spacetime. This naturally suggests extensions of the present strategy to these fields, which I outline, leaving the details, however, for future work.

\section{The Strong Equivalence Principle}

There is a venerable tradition of considering the relationship between the special and general theories of relativity in terms of an equivalence principle (Pauli, 1958). Although the equivalence principle is rightly attributed to Einstein, he took the principle rather to be a covariance principle applied in Minkowski spacetime only, which allowed one to interchange uniformly accelerated frames with unaccelerated ones in a uniform gravitational field (Norton, 1993, §4.1); he did not see it as linking the special and general theories (Einstein, 1923, 1956). Advocates of such a link have acknowledged this (Read et al, 2018, p. 14n2), emphasizing that one should understand the relevant version as the strong equivalence principle (SEP), rather than Einstein's equivalence principle. Here is a typical statement by Brown (2005, p. 169):

There exists in the neighborhood of each event preferred coordinates, called locally inertial at that event. For each fundamental non-gravitational interaction, to the extent that tidal gravitational effects can be ignored the laws governing the interaction find their simplest form in these coordinates. This is their special relativistic form, independent of space-time location.

This raises at least three interpretive questions:

1. What, exactly, are laws governing fundamental non-gravitational interactions?

2. What does it mean that "tidal gravitational effects can be ignored"? 
3. What is does it mean for a law to take "special relativistic form," or its "simplest form"?

Read et al (2018, p. 17) clarify further each of these in their restatement of the SEP: "The dynamical equations for non-gravitational fields reduce to a Poincaré invariant form, with no terms featuring the Riemann tensor or its contractions, in a neighborhood of any $p \in M$." Thus they provide the following answers:

1. The laws governing fundamental non-gravitational interactions are the dynamical equations for matter fields, those fields with an associated energy-momentum tensor (Read et al, 2018, p. 14n1).

2. Tidal gravitational effects are terms in the aforementioned dynamical equations that make explicit reference to curvature; justifiably "ignoring" them requires sufficiently low sensitivity of one's experimental apparatus to curvature effects that they cannot differentiate between the presence and absence of curvature (Read et al, 2018, p. 17); thus this clause can only be satisfied contextually (Brown, 2005, p. 170).

3. An equation takes special relativistic form when it is invariant under Poincaré transformations; it takes its simplest form when the number of terms in the equation does not reduce by being expressed in a special local inertial frame (Read et al, 2018, p. 21).

I will return to further elucidation of the third answer shortly; regarding the second answer, Brown (1997, p. 72) emphasizes the context also includes the size of the region under consideration-a region $U_{\mathcal{E}}(p)$ containing $p$ where one can find the aforementioned locally inertial coordinates. These are coordinates in which the connection components within $U_{\mathcal{E}}(p)$ are sufficiently small that, for geodesic worldlines of free point particles intersecting this region, their coordinate expressions do not measurably deviate from straightness. (In other words, their coordinate velocities are approximately constant.) So, when Brown (1997, p. 71) writes that "In GR, for all regions of space-time in which curvature can be ignored, SR is valid by fiat," the decree to which he alludes is the SEP as stated above.

Does Brown's claim about the local validity of SR include spacetime symmetries? Some of his comments suggest that it should:

It should be noted also that with respect to $U_{\mathcal{\varepsilon}}(p)$ it is perfectly legitimate to define "local" symmetry groups that contain as a sub-group the set of spatial translations, such as the Poincare group in SR. This calls into question the frequent claim that it is the Lorentz group, rather than the Poincaré group, which represents the local symmetries in GR. (Brown, 1997, p. 79n17)

However, here we must return to the third answer above to adjudicate whether the local validity of SR, as the SEP purportedly grants, guarantees the existence of local Poincaré spacetime symmetries. As Read et al (2018, p. 19) explain, a problem arises with their interpretation, if they were to be taken as spacetime symmetries properly understood: on the one hand, for any event $p$ SR is supposed only to be valid in some $U_{\varepsilon}(p)$, yet the Poincaré group contains actions (such as translations) that seem like they ought to map $p$ to a point $q$ outside of $U_{\mathcal{E}}(p)$; thus understood, some spacetime symmetries are not symmetries applicable to $U_{\varepsilon}(p)$ at all. 
Put plainly, the symmetries of $U_{\mathcal{E}}(p)$ cannot be the Poincare symmetries because that group is not even well-defined on a bounded region of spacetime. "The resolution is to view the Poincaré transformations discussed above as passive only- their action is not on spacetime points at all, but rather on the chart space, i.e., the codomain of the coordinate charts" (Read et al, 2018, p. 19n32). In other words, the normal (and "locally inertial") coordinate system assigned to $U_{\mathcal{E}}(p)$ to which the SEP refers is a fragment of a Lorentz chart, and the Poincaré transformations invoked act on these charts, shifting the coordinates assigned to points of $U_{\mathcal{E}}(p)$ to one related by the invoked Poincaré transformation. No diffeomorphisms or pushforwards are invoked that would map points of $U_{\mathcal{\varepsilon}}(p)$ outside it, but this is not needed to account for the invariance of form of equations referring only to points of $U_{\mathcal{E}}(p)$.

This makes the invocation of the SEP for its stated aims salutary and consistent. ${ }^{6}$ But it doesn't explain or account for local spacetime symmetries. Those do not concern directly the preservation of forms of equations, which is a mostly syntactic notion, but rather the invariance of spacetime structure, which is a mostly semantic notion. Moreover, as I show in section 3.2, the SEP holding approximately on a spacetime region, to any given degree of approximation, is neither necessary nor sufficient for that region to have even approximate local Poincaré spacetime symmetry.

One possible reply to this conundrum would be to re-define what it means for a theory to be "special relativistic." Read et al $(2018, \S 7)$ describe, but do not advance, one way to do this: take such theories to be characterized solely by the constraint that "The dynamical laws governing matter fields are Poincaré invariant," instead of also assuming that "The inertial frames are global" (Read et al, 2018, p. 22), i.e., that the covariant connection is flat (and arises from a geodesically complete metric on $\mathbb{R}^{4}$ (Read et al, 2018, p. 22n45)). The advantage they cite for this position is that it allows one to claim that SR is locally valid exactly, instead of merely approximately.

But this is a doubly Pyrrhic victory. By moving the goalposts so much closer, it elides much of why the local validity of SR was important in the first place. The point was never to find an interpretation of the sentence "SR is locally valid" that makes it true, but rather to explain why SR is as successful as it is, despite its idealizations, on its own terms. SR as such employs Minkowski spacetime, with full Poincaré symmetry, and the conservation laws that this entails. Re-construing SR does not explain the successful albeit approximate application of these symmetries to local regions. Moreover, it conceals the important insight that the extent of SR's

\footnotetext{
${ }^{6}$ That said, it does bring out a lacuna in the argument for the chronogeometric significance of the metric - the argument for why, according to the dynamical perspective on relativity theory (Brown, 1997; Brown and Pooley, 2001; Brown, 2005), the spacetime metric (perhaps only approximately) measures or surveys times and distances. The argument hinges on observing that "The symmetries of the dynamical laws governing non-gravitational fields in the appropriate local neighborhood ...coincide with the symmetries of the dynamical metric field in this neighborhood" (Read et al, 2018, p. 19), with the latter understood as spacetime symmetries in the sense I have discussed (Read et al, 2018, p. 19n25). While the symmetry groups coincide, they act on different objects: the former acts on coordinates assignments to points and fields in a fixed region, while the latter acts on spacetime points and fields thereon. Why should this coincidence of two different types of objects deliver the interpretation of one?
} 
success is based on approximation, not exact correspondence, and is limited in general to a neighborhood of an event. Without this insight, it becomes entirely obscure why and when SR is in certain circumstances empirically inadequate in comparison to GR.

\section{Poincaré Symmetry}

In the previous section, I invoked general features of approximate spacetime symmetries qua spacetime symmetries in my argument that they are not adequately captured by the SEP. But I had not yet given a precise definition of what it means for a symmetry to be local, or-more importantly-approximate. That is the task of the first subsection, 3.1. The second subsection, 3.2, will introduce examples showing that Poincare invariance of equations, as invoked by the SEP, is neither necessary nor sufficient for Poincaré spacetime symmetry.

\subsection{Local and Approximate Spacetime Symmetries}

In section 1.2, I introduced a spacetime symmetry of a spacetime $(M, g)$ as an element of a collection of diffeomorphisms $\psi$ on $M$ such that $\psi_{*}(g)=g$, and which form a group under composition. While this definition is slightly more general than is usually found in textbook treatments, which typically focus on symmetries that are generated as the flows of Killing vector fields (Malament, 2007, §2.7) or fields that satisfy generalizations of Killing's equation (Wald, 1984, Ch. C.3), it is also not general enough for present concerns in two ways. First, as the action of $\psi$ is on all of $M$, it is a global symmetry, while an account of local symmetries on just proper parts of $M$ is needed. Second, the condition that the action of the diffeomorphism preserves the metric, $\psi_{*}(g)=g$, is exact, while an account of approximate preservation is needed. These will be taken up and then combined in the next two subsections.

\subsubsection{Local Spacetime Symmetries}

The modification of the definition of global spacetime symmetries to yield that of local spacetime symmetries is quite simple. Let a spacetime $(M, g)$ be given, and $U, V$ be open submanifolds of $M$. The smooth map $\psi: U \rightarrow V$ is said to be a local diffeomorphism when it is a diffeomorphism of $U$ considered as a manifold in its own right. If further $\psi_{*}(g)=g$, i.e., the pushforward of the metric on $U$ along $\psi$ yields the metric on $V$, then $\psi$ is a local spacetime symmetry of $(M, g)$ (Hall, 2004, p. 285). When $U=M, \psi$ is also a global spacetime symmetry, but this is clearly a special case. For instance, consider any global spacetime symmetry of Minkowski 
spacetime, e.g., a time translation; when restricted to some open bounded (i.e., precompact) region, it is a local spacetime symmetry. If one transforms the Minkowksi metric by, say, a conformal factor that is not constant outside of the domain and range of this local spacetime symmetry, it still remains a local spacetime symmetry, even though after making this transformation there are no non-trivial global spacetime symmetries.

The collection of all local spacetime symmetries for $(M, g)$ does not typically form a group under composition because these symmetries in general do not share domains and codomains. However, whenever $\psi: U \rightarrow V$ and $\psi^{\prime}: U^{\prime} \rightarrow V^{\prime}$ are local spacetime symmetries and $V \subseteq U^{\prime}$, then $\psi^{\prime} \circ \psi$ is also a local spacetime symmetry. In other words, the collection is closed under composition when the composition is well-defined. Moreover, the identity map on $M$ counts as a local diffeomorphism, and because each local spacetime symmetry is a diffeomorphism of its domain, its inverse is a local spacetime symmetry as well. This yields a slight generalization of the group concept known as a groupoid. Thus, we may speak of the groupoid of all local spacetime symmetries for $(M, g)$.

Local spacetime symmetries can be generated infinitesimally from smooth vector fields, just as global spacetime symmetries can, and give rise to conserved quantities in much the same way. The smooth vector field generating such a local symmetry for a region is called a local Killing vector field for that region. It must be defined at least on a larger region connecting the domain of the putative symmetry with its range. One important difference between local and global symmetries, however, is that the parameter for the flows that these local Killing vector fields generate may only be defined for a proper subinterval of $\mathbb{R}$. For example, in a spacetime describing gravitational collapse into a Schwarzschild-like black hole (Wald, 1984, p. 155-7), once the collapse has finished there is a local timelike (and hypersurface orthogonal) Killing vector field, i.e., the spacetime is locally static (Malament, 2007, p. 253). But that Killing vector field does not extend into the past, i.e., to the portion of spacetime during and before the collapse process.

Given a collection of local Killing vector fields for a region, one can classify what sort of spacetime symmetry group they model by examining the Lie algebra of the fields as they are defined on that region. Recall that the Lie bracket for the Lie algebra of such fields is defined through the Lie derivative, i.e., for vector fields $\alpha, \beta, \gamma$ defined on a common region $U$, their bracket is defined as $[\alpha, \beta]=f_{\alpha} \beta$, which is anti-symmetric $([\alpha, \beta]=-[\beta, \alpha])$ and satisfies the Jacobi identity,

$$
[[\alpha, \beta], \gamma]+[[\gamma, \alpha], \beta]+[[\beta, \gamma], \alpha]=0 .
$$

Moreover, the Lie bracket of local Killing vector fields on a region is a local Killing vector field on the same region. So, a region may be said in particular to have local Poincaré spacetime symmetry when there is a collection of local Killing vector fields on that region whose Lie algebra is the Poincaré algebra. ${ }^{7}$

\footnotetext{
${ }^{7}$ I am eliding some inconsequential technicalities regarding the relationships between local diffeomorphisms, local transformation groups (associated with a connected Lie group), and infinitesimal
} 


\subsubsection{Approximate Spacetime Symmetries}

An approximate (local) spacetime symmetry $\psi$ should be a (local) diffeomorphism that satisfies the equation $\psi_{*}(g)=g$ approximately. To make sense of this demand, one must describe how a spacetime metric_-or even a tensor field, more generallyapproximates or is similar to another on a spacetime region. There is good reason to believe that there is no canonical way to do this, so we must determine from the present context of investigation what the relevant notion of similarity is (Fletcher, 2016). Although local symmetries may be defined on arbitrary submanifolds, I focus here on bounded (i.e., precompact) regions only. This restriction fits with the present goals because the ultimate explanandum here is the successful application of the spacetime symmetries of SR, which has been only in bounded regions (but much more than isolated points) of spacetime.

In this case, one can adapt the apparatus of the so-called compact-open topologies on Lorentz metrics, as developed in Fletcher (2014). To begin with, consider a precompact region $U$ on which one is adjudicating the status of a putative collection of local spacetime symmetries. Next, consider a smooth frame field $\left\{t^{a}, x^{a}, y^{a}, z^{a}\right\}$ defined at least on the closure of the union of the images of $U$ under those symmetry maps, recalling that a frame field is an collection of orthonormal vector fields, one of which $\left(t^{a}\right)$ is timelike and the rest spacelike, that form a basis for the tangent space at any point. Such a frame field represents idealized temporal and spatial measuring instruments, and each of its component's local integral curves are curves of constant temporal or spatial coordinate value. From it, one can construct the smooth (inverse) Riemannian metric ${ }^{8}$

$$
h^{a b}=t^{a} t^{b}+x^{a} x^{b}+y^{a} y^{b}+z^{a} z^{b},
$$

which in turn defines a norm-what I will call the $h$-fiber norm-for covariant tensor fields, such as Lorentz metrics $g_{a b}$, at points where it is defined:

$$
|g|_{h}=\left|h^{a b} h^{c d} g_{a c} g_{b d}\right|^{1 / 2} .
$$

Inspection shows that the $h$-fiber norm of $g$ at a point returns the Frobenius norm of $g$ expressed as a matrix in components of the originally chosen frame field, i.e., the square root of the sum of squares of these components. It serves as an aggregate measure of the magnitude of a field at a point as measured using the frame field. Furthermore, the definition of the $h$-fiber norm can be extended to fields with arbitrary covariant indices, $f_{a_{1} a_{2} \cdots a_{n}}$ as follows:

$$
|f|_{h}=\left|h^{a_{1} b_{1}} h^{a_{2} b_{2}} \cdots h^{a_{n} b_{n}} f_{a_{1} a_{2} \cdots a_{n}} f_{b_{1} b_{2} \cdots b_{n}}\right|^{1 / 2} \text {. }
$$

\footnotetext{
transformation groups (associated with a Lie algebra). For more on these, including references, see Hall (2004, Ch. 5.11).

${ }^{8}$ In this mode of presentation, I have assumed that the region in question is temporally and spatially orientable, for this is equivalent to the existence of a frame field. (See footnote 11 for definitions of these properties.) However, even if the region did not have those properties one can always start with some smooth (inverse) Riemmanian that can be decomposed locally into a frame field.
} 
The significance of this extension is that it allows us to define a distance function between two metrics on a region $U$ that compares the maximal differences not only of their components as expressed in the basis of the frame field, but also their derivatives up to order $k$ :

$$
d_{U}\left(g, g^{\prime} ; h, k\right)=\max _{j \in\{0, \ldots, k\}} \sup _{U}\left|\nabla^{(j)}\left(g-g^{\prime}\right)\right|_{h},
$$

where $\nabla^{(j)}\left(g-g^{\prime}\right)$ abbreviates $\left(g_{a b}-g_{a b}^{\prime}\right)$ for $j=0$ and $\nabla_{c_{1}} \cdots \nabla_{c_{j}}\left(g_{a b}-g_{a b}^{\prime}\right)$ otherwise, with $\nabla$ the Levi-Civita connection compatible with $h$.

With this apparatus in place, one can define an approximate (local) spacetime symmetry $\psi$ on $U$. More precisely, a local diffeomorphism $\psi: U \rightarrow V$ (of course with $U, V \subseteq M)$ is an $(h, \varepsilon)$-spacetime symmetry to order $k$ on $U$ when $d_{U}\left(g, \psi^{*}(g) ; h, k\right)<\varepsilon$. (Note that when $\psi$ is a member of a one-parameter family of local diffeomorphisms generated by a local Killing vector field $\kappa$, this is equivalent to the condition that $\sup _{U}\left|f_{K} \nabla^{(j)} g\right|_{h}<\varepsilon$.) In other words, the maximum difference between the metric on $U$ and the metric on the image of $\psi$, including its derivatives to order $k$, is no more than $\varepsilon$ according to the frame field constructing $h$. More generally, one may say that $U$ has an $(h, \varepsilon)$-spacetime symmetry groupoid $G$ to order $k$ on $U$ when $\sup _{\psi \in G} d_{U}\left(g, \psi^{*}(g) ; h, k\right)<\varepsilon$ and the elements of $G$ form a groupoid. Further, when they are members of a one-parameter family of local diffeomorphisms generated by local Killing vector fields, one can classify this groupoid by the Lie algebra of the vector fields formed under the Lie bracket.

Theorem 1. For every $h, \varepsilon$, and finite $k$ as above, every point of every spacetime has a neighborhood on which there is $(h, \varepsilon)$-Poincaré spacetime symmetry to order $k$, where the usual group of symmetries is restricted to a groupoid that forms a neighborhood of the identity in the Poincaré Lie group.

Proof. Consider any spacetime $(M, g)$, any event $p \in V \subseteq M$, and a diffeomorphism $\phi: U \rightarrow V$, where $U \subseteq \mathbb{R}^{4}$ is a neighborhood of Minkowski spacetime $\left(\mathbb{R}^{4}, \eta\right)$ such that $\phi_{*}(\eta)_{\mid p}=g_{\mid p}$. (It is always possible to satisfy this last condition because any tangent space at any point of each spacetime, equipped with a Lorentz metric, is isomorphic as an inner product space to any other such.) Consider also any Riemannian $h$ defined at least on $U$, any $\varepsilon>0$, and any finite non-negative $k$. One can push forward onto $V$ a collection of local Killing vector fields on $U$ that form a basis for the Poincaré algebra. Each of these generates a one parameter family of local flows $\psi_{t}$ with flow parameter $t$. Since $g$ is smooth, one can always find $V^{\prime} \subseteq V$ that is a neighborhood of $p$ and some $t^{\prime}>0$ such that for all $|t|<t^{\prime} d_{V^{\prime}}\left(g, \psi_{t}^{*}(g) ; h, k\right)<\varepsilon / 10$. Since the Poincare algebra is ten-dimensional, any linear combination of these local Killing vector fields with coefficients no greater than one will then generate a local flow $\tilde{\psi}_{t}$ such that $d_{V^{\prime}}\left(g, \tilde{\psi}_{t}{ }^{*}(g) ; h, k\right)<\varepsilon$ for sufficiently small $t$, using the triangle inequality and the linearity properties of the Lie derivative. These linear combinations in turn, through the Lie exponential map, generate elements of the Poincaré Lie group in its identity component, which form a groupoid. Finally, because $\phi_{*}(\eta)_{\mid p}=g_{\mid p}$, these elements can be interpreted as translations, rotations, 
boosts, and combinations thereof, according to the corresponding interpretations of the elements of the Poincaré Lie group for Minkowski spacetime.

It remains to show that the elements of this groupoid satisfy the commutation relations of the Poincare algebra on $V^{\prime}$. For this, it suffices to prove that, if $\alpha$ and $\beta$ are smooth vector fields on $U$, then $\phi_{*}([\alpha, \beta])=\left[\phi_{*}(\alpha), \phi_{*}(\beta)\right]$. For in this case, it follows from any commutation relation $[\alpha, \beta]=\gamma$ for smooth vector fields on $U$ that $\phi_{*}(\gamma)=\phi_{*}([\alpha, \beta])=\left[\phi_{*}(\alpha), \phi_{*}(\beta)\right]$ on $V$, hence on $V^{\prime}$.

The proof requires the application of three facts: for any smooth vector fields $\xi, \zeta$ and smooth scalar field $f$ on $U$,

$$
\begin{gathered}
{[\xi, \zeta](f)=\xi(\zeta(f))-\zeta(\xi(f)),} \\
\phi_{*}(\xi)(f)_{\mid p}=\xi(f \circ \phi)_{\mid \phi^{-1}(p)}, \\
\phi_{*}(\xi)(f) \circ \phi=\xi(f \circ \phi) .
\end{gathered}
$$

Equation 6 expresses the action, on a smooth scalar field, of the Lie bracket of smooth vector fields in terms of the commutator of directional derivatives of that scalar field. Each of equations 7 and 8 expresses two different ways of writing the pushforward of a smooth vector field, acting on a scalar field, at points of $M$ or $\mathbb{R}^{4}$, respectively. Thus, letting $\alpha$ and $\beta$ be smooth vector fields on $U, p \in U$, and $f$ be a smooth scalar field on $V$,

$$
\begin{aligned}
\phi_{*}([\alpha, \beta])(f)_{\mid p} & =[\alpha, \beta](f \circ \phi)_{\mid \phi^{-1}(p)} \\
& =\alpha(\beta(f \circ \phi))_{\mid \phi^{-1}(p)}-\beta(\alpha(f \circ \phi))_{\mid \phi^{-1}(p)} \\
& =\alpha\left(\phi_{*}(\beta)(f) \circ \phi\right)_{\mid \phi^{-1}(p)}-\beta\left(\phi_{*}(\alpha)(f) \circ \phi\right)_{\mid \phi^{-1}(p)} \\
& =\phi_{*}(\alpha)\left(\phi_{*}(\beta)(f)\right)_{\mid p}-\phi_{*}(\beta)\left(\phi_{*}(\alpha)(f)\right)_{\mid p} \\
& =\left[\phi_{*}(\alpha), \phi_{*}(\beta)\right](f)_{\mid p} .
\end{aligned}
$$

Equations 9 and 12 apply equation 7; equations 10 and 13 apply equation 6; equation 11 applies equation 8 . Since $f$ and $p$ were arbitrary, equation 13 holds generally.

Before moving on, a note of comparison is in order regarding the sense in which approximate symmetries are observer-dependent. Applications of the SEP as discussed above are observer-dependent in the sense that they depend on the experimental apparatus available to measure "the strength of curvature effects" (Read et al, 2018 , p. 17) that would distinguish solutions to an SR dynamical equation from those to a GR one in which curvature appears. These apparatus are also considered to be bounded, generally: "Whether you can detect tidal effects in a space the size of the room [you are in] depends on what kind of equipment you have access to, or in some cases how much time you have at your disposal!" (Brown, 2005, p. 170). By contrast, for approximate spacetime symmetries, there is no simple connection with curvature. On the one hand, it may be possible for curvature effects, insofar as they are coded in the second derivatives of the metric, to be quite strong in magnitude, yet the metric on the image of the local symmetry map is quite similar, so that their difference is quite small and does not preclude approximate symmetry for a fixed 
pair $(h, \varepsilon)$. On the other, it may be possible for curvature effects to be quite small, yet for the differences in higher or lower (than the second) derivatives of the metric to be substantial enough to preclude approximate symmetry for $(h, \varepsilon)$.

\subsection{Poincaré Invariance of Equations and Poincaré Spacetime Symmetry}

One question that arises naturally from the foregoing is what logical relationship the Poincaré invariance of equations and Poincaré spacetime symmetries have with one another in relativistic spacetimes. In this subsection, I show that the one holding of a certain spacetime region does not imply the other on the same region. The examples are fairly simple and illustrate how the two concepts come apart.

\subsubsection{Poincaré Spacetime Symmetry without Poincaré Invariance of Equations}

Here I adopt a simple example from Read et al (2018, p. 24n51). It is special relativistic, thus set in Minkowski spacetime $\left(\mathbb{R}^{4}, \eta\right)$, which has not just approximate but exact Poincaré spacetime symmetry globally. However, it does not have Poincaré invariance of its dynamical equations in their simplest form. ${ }^{9}$

Consider a dust field with positive density $\rho$ whose four-velocity field $v^{a}$ forms a geodesic congruence. Its energy-momentum tensor is simply $T_{a b}=\rho v_{a} v_{b}$, and its dynamical equations follow from the conservation condition $\nabla_{a} T^{a b}=0$ (Malament, 2007, p. 243):

$$
\begin{aligned}
v^{b} \nabla_{b} v^{a} & =0, \\
v^{b} \nabla_{b} \rho+\rho\left(\nabla_{b} v^{b}\right) & =0 .
\end{aligned}
$$

The first, equation 14, is just the geodesic equation for the dust's worldlines. The second, equation 15, is a kind of energy conservation condition: its first term describes the change in energy density while its second the change in relative volume, both as described by a co-moving observer.

Thus it is not surprising that the equations of motion simplify in the relevant sense when expressed in coordinates well-adapted to such a co-moving frame. In particular, one can find such a frame in whose adapted coordinates all the spatial components of $v^{a}$ vanish, i.e., $v^{\mu}=0$ for $\mu=1,2,3$. Furthermore, the temporal component is constant, i.e., $v^{0}=1$ assuming normalization using geometric units.

\footnotetext{
${ }^{9}$ Here I follow Read et al (2018, p. 21) in construing one form to be simpler than another if it contains fewer terms. Although I am skeptical of the cogency of this notion-cf. my similar remarks about the hyperintensionality of minimal coupling in the concluding section 5-insofar as it undergirds the definition and application of Poincaré invariance, which frames the question of its relation to Poincaré spacetime symmetry, I adopt it for those purposes without broader endorsement.
} 
Thus equations 14 and 15 simplify to a single equation:

$$
v^{0} \nabla_{0} \rho=0
$$

stating that the density is constant along any integral curve of $v^{a}$. Clearly, the form of this equation is not preserved under Poincaré transformations of the chart in which it is expressed. Effectively, the four-velocity of the dust determines a preferred frame.

It might be objected that in order to count, the new field must not contribute to the energy-momentum tensor but rather be a part of spacetime structure or a fixed field of some sort. ${ }^{10}$ In that case, one can introduce any timelike geodesic congruence $\tau^{a}$ separately to define a collection of preferred frames, and express the equations of motion of all matter fields in terms of it. Requiring that any dust field be spatially homogeneous with respect to coordinates adapted to these frames, which only differ by spatial rotations, results in the simplification of equation 15 to

$$
v^{0} \nabla_{0} \rho+\rho\left(\nabla_{\mu} v^{\mu}\right)=0 .
$$

For further examples, see Carroll and Lim (2004), Jacobson and Speranza (2015), and references therein.

\subsubsection{Poincaré Invariance of Equations without Poincaré Spacetime Symmetry}

The claim that it's possible to have Poincaré invariance of equations without even approximate Poincaré spacetime symmetry may seem at first in tension with the main results of section 3.1, namely that every spacetime has approximate local Poincaré spacetime symmetry. One can resolve this tension through careful attention to the logical form of the definitions. Recall that a spacetime having a local symmetry just means that every point of the spacetime has a neighborhood that is the domain of a symmetry. But this does not entail that every neighborhood of a point is such a domain. Indeed, in a relativistic spacetime a generic neighborhood will not have even approximate Poincaré spacetime symmetry. Thus one way that a region could have approximate Poincaré invariance of equations applied within it but not approximate Poincaré spacetime symmetry would be if the approximation criteria for the former did not entail the latter. Whether this is so is not entirely clear because it's not clear what the relationship between fields in a spacetime approximately being solutions to a certain equation and that spacetime having approximate symmetries is. However, the discussion at the end of section 3.1 about the mismatch between approximation of the metric and its derivatives by another and the small magnitude of curvature effects suggests a lack of entailment.

In any case, there are also topological constraints that can prevent a region from having Poincaré symmetry without precluding the Poincaré invariance of the equa-

${ }^{10}$ I take this to be a plausible reading of discussions in Brown (2005, p. 171) or Read et al (2018, p. 24n52), which suggest some criterion like this as mandatory. 
tions for matter fields on that region. Consider again Minkowski spacetime, $\left(\mathbb{R}^{4}, \eta\right)$, and a Lorentz chart $\phi: \mathbb{R}^{4} \rightarrow \mathbb{R}^{4}$ whose domain is the whole manifold of the spacetime. For any dynamical equations for fields on it, if they are Poincaré invariant for the whole spacetime manifold, then they are so for any proper submanifold. So for any point of the manifold $p \in \mathbb{R}^{4}$, those equations are Poincare invariant for the spacetime $\left(\mathbb{R}^{4}-\{p\}, \eta_{\mid \mathbb{R}^{4}-\{p\}}\right)$. But that whole spacetime cannot have Poincaré spacetime symmetry, simply because as a spacetime region it is the wrong topology to support such symmetry. What were the Poincaré symmetry Killing vector fields on Minkowski spacetime are no long Killing vector fields, because any non-trivial flow along them is no longer well-defined. (They flow "into" the point removed.)

This can be the case even if the region considered is diffeomorphic to $\mathbb{R}^{4}$. Just as the dynamical equations that are Poincaré invariant on Minkowksi spacetime are still Poincaré invariant on Minkowksi spacetime sans an arbitrary point $p$, they are also Poincaré invariant on any region not containing $p$ but with $p$ in its closure. Such a region, considered now in the spacetime $\left(\mathbb{R}^{4}-\{p\}, \eta_{\mid \mathbb{R}^{4}-\{p\}}\right)$, also cannot have Poincaré spacetime symmetry, for any putative Killing vector field on the region cannot be extended to a vector field that generates even a local one-parameter group of diffeomorphisms acting on the region.

Yet another further obstruction to Poincaré spacetime symmetry on a region arises when some putative Killing vector field on the region cannot be extended smoothly to a vector field that generates even a local one-parameter group of diffeomorphisms acting on the region. This can occur when the union of the images of the diffeomorphisms are not time- or space-orientable, even if their domain is. ${ }^{11}$ For a simple two-dimensional example, consider the unit square with two of its boundary edges, $(0,1) \times[0,1]$, identifying the edges after a twist and removing the two resulting boundary points to form a Möbius band $M$ (i.e., a Möbius strip without boundary). Label the now identified edges (sans two boundary points) as region $E$, and consider any flat Lorentz metric on $M$. The region $M-E$ is isometric to a simplyconnected region of Minkowski spacetime. Thus, any dynamical equations for fields on that region are Poincaré invariant. But no putative set of Killing vector fields on $M-E$ generating Poincaré spacetime symmetries can be extended smoothly onto E.

\section{Local Approximate Geometry}

One of the examples from section 3.2 sheds light on contrasting positions on the role of "local approximate spacetime geometry" in Torretti (1996) and Brown and Pooley (2001). To frame this contrast, recall that, in his interpretation of Schrödinger (1950), Brown (1997, p. 68) writes that "It is a fundamental assumption in GR that

\footnotetext{
${ }^{11}$ Recall that a spacetime is time-orientable when there exists a continuous classification of timelike vector fields on the spacetime into future- and past-directed; it is space-orientable when there exists a continuous classification of orthogonal spacelike vector field triads on the spacetime into left- and right-handed (Wald, 1984, p. 60).
} 
the local structure of space-time, suitably defined, is special relativistic." Brown (1997, p. 71) has in mind in particular that "relative to local inertial frames (defined in the infinitesimal neighborhood of any event) all the laws of physics take on their special relativistic form. Put another way, the tangent space structure in GR is everywhere 'Lorentzian'." These paired claims, the first an expression (as discussed in section 2) of the SEP and the second about spacetime geometry, form the basis for a putative explanation of SR's success (or, at least, how he takes this to be assumed in the formulation of GR). ${ }^{12}$

On one interpretation, this is what Torretti (1996, p. 136) argues, too, for according to him,

no effect of gravity will be disclosed—within the agreed margin of precision — by any description of natural phenomena in terms of [the coordinates of a local Lorentz chart]; and that the laws of nature take the same form in [that neighborhood], when referred to [those coordinates], as they would referred to an ordinary Lorentz chart in a spacetime region where gravity is absent. ... [This] implies that two experiments whose initial conditions read alike in terms of [local Lorentz charts] will also have the same outcome in terms of these charts. ${ }^{13}$

This corresponds with the first claim. For the second, he writes further that "the assumption that [the spacetime metric in GR] has the same signature as the Minkowski metric $\eta$ is itself empirically motivated, insofar as it entails that every tangent space of the manifold is isometric with Minkowski spacetime, and thus accounts for the local success of Special Relativity" (Torretti, 1996, p. 139). ${ }^{14}$ More precisely, "The Minkowski inner product on each tangent space induces - through the exponential mapping - a local approximate Minkowski geometry on a small neighborhood of each worldpoint. This accounts for the Lorentz invariance of the laws of nature referred to local Lorentz charts" (Torretti, 1996, p. 240). Thus, initially it seems that Brown and Torretti are in agreement regarding the explanatory nature of the paired claims and their status as assumptions, although Torretti does not equate the two claims but asserts rather that the first claim-about the laws of physics-follows from the second-about approximate local spacetime geometry.

However, Brown and Pooley (2001, p. 270) later deny this implication (hence implicitly Brown's earlier expression of the synonymy of these two expressions), insisting that laws involving the dynamics of matter fields must additionally satisfy the so-called minimal coupling condition for the former expression to be true:

\footnotetext{
${ }^{12}$ Astute readers may wonder just how the second claim is supposed to be a paraphrase of the first. This will play a role in the emerging dispute and its resolution, below.

13 To make this last statement, Torretti (1996, p. 54) also invokes Einstein's "Principle of Relativity," that "The laws by which the states of physical systems undergo change are not affected, whether these changes of state be referred to the one or to the other of two Lorentz charts."

${ }^{14}$ It's important to distinguish Minkowski spacetime, which, having no preferred origin, has at most the structure of an affine space, from the tangent space of a relativistic spacetime at a pointwhat Hall (2004, p. 147) calls Minkowski space - which does have a preferred origin and thus has the structure of a vector space. Charitably, then, by "isometric with Minkowski spacetime" Torretti means either "isometric with that of any point of Minkowski spacetime" or, what is essentially equivalent, "isometric with Minkowski space."
} 
In our view, this claim [by Torretti] is a non sequitur. It is mysterious to us how the existence of a local approximate Minkowski geometry entails the Lorentz covariance of the laws of the non-gravitational interactions. Theories postulating a Lorentzian metric but which violate minimal coupling would involve non-Lorentz covariant laws. ... It seems to us that the local validity of special relativity in GR cannot be derived from what Torretti takes to be the central hypothesis of GR above, but must be independently assumed.

A matter theory is said to be minimally coupled when one arrives at the dynamical equations for its fields in GR by performing the following substitution procedure on its dynamical equations in SR: replace all instances of the Minkowski metric with a general Lorentzian metric, and all instances of the flat covariant connection with the Levi-Civita connection. ${ }^{15}$ Thus, according to Brown and Pooley (2001), the first claim above - an expression of the SEP — is in fact independent of the second claim-about spacetime geometry—and so must be an additional assumption in the explanation of the success of SR.

Later, Brown (2005, p. 170-1) implicitly takes back the justification for this claim, that the reason the SEP does not follow from features of the local geometry of spacetime is that this geometry is compatible with non-minimally coupled matter fields, and non-minimally coupled matter fields have non-Lorentz invariant dynamical laws. He acknowledges instead that the dynamical equations for nonminimally coupled fields may still be Lorentz invariant. But then what sort of counterexample is supposed to confound Torretti's claim? Here is where the example of a non-Lorentz invariant matter theory on a relativistic spacetime comes in. Recall that this example was not invariant in the sense described precisely because there are a class of preferred frames in which the dynamical equations simplify. In other words, the definition of their simplest forms adverts to a spatiotemporal quantity or direction that is not derived from matter fields-it does not contribute to the energymomentum tensor-and so must be a part of spacetime structure beyond the metric.

How does this example bear on Torretti's claim above, that the "local approximate Minkowski geometry on a small neighborhood of each worldpoint ... accounts for the Lorentz invariance of the laws of nature referred to local Lorentz charts" (Torretti, 1996, p. 240)? If by "laws of nature referred to Lorentz charts" Torretti meant merely laws that use Lorentz coordinates, this would indeed be a counterexample to his claim. However, in later writing Torretti (1999, p. 283n) clarifies his position with an example:

the requirement of Lorentz invariance [imposed by SR] holds only for physical laws referred to a Lorentz chart. Therefore, the requirement cannot properly apply to Newton's laws, for the time variable that appears in them is not Einstein time. ... if one charitably replaces it with Einstein time, the Laws thus refurbished are not Lorentz invariant.

Here, "Einstein time" is simply the time variable that coordinatizes a Lorentz chart, while the time coordinate appearing in Newton's laws adverts to an observerindependent (i.e., observer-invariant) temporal structure. The other, spatial variables

15 Actually, Brown (2005, p. 170) states that minimal coupling involves the non-appearance of terms depending on spacetime curvature in the dynamical equation, but Read et al $(2018, \S 3.2)$ rightly point out that this is in general not true, even accounting for the ambiguities of the application of the minimal coupling prescription as I have described it. 
that appear in the laws, meanwhile, are those that normally coordinatize a Lorentz chart. Thus, in his (1996) Torretti was in fact referring to laws that advert only to Lorentz coordinates. Examples like the non-Poincaré-invariant one from section 3.2 are excluded because they refer to other spacetime structure that defines other coordinates. So, ultimately the criticism is evaded: that the local geometry of spacetime is approximately Minkowskian in the sense described does entail the Lorentz invariance of dynamical laws that only advert to the metric spacetime structure, hence only to Lorentz chart coordinates (Hall, 2004, pp. 286-7). It is after all these sorts of dynamical laws that are in play in SR, and the ones whose successful application is to be explained. The goal is not to recover (however approximately) laws not actually used in SR, but only those actually successfully applied; these laws are never excluded from Torretti's claim.

\section{Conclusions, and Prospects for a Broader Explanation of the Success of SR}

As I described in section 1, the questions of the "local validity" of SR in GR ought to be interpreted as a question about reduction and explanation: GR reduces to SR locally, meaning that it explains the successful applications of SR within the bounds of allowable approximation. And one aspect of this explanation is the local application of spacetime symmetries, with their concomitant conserved quantities, in spacetimes that need have no global symmetries whatsoever. While the literature concerning the SEP may seem to address this question, I argued in section 2 that in fact it does not; it focuses instead on the forms of dynamical laws and their invariance under Poincaré coordinate transformations, not Poincaré spacetime symmetries. I showed that neither of the two implies the other in section 3.2, after describing in some detail my positive account of local approximate spacetime symmetries in section 3.1, and how in particular every spacetime has approximate local Poincaré spacetime symmetries. Finally, in the previous section (section 4), I applied these examples and a close reading of Torretti's account of the explanation of the success of SR to exonerate him from the charge of committing a non sequitur.

One issue raised in that last section was the role of "minimal coupling" in accounting for the invariance of the dynamical equations of matter fields under Poincaré transformations. Recall that minimal coupling is a prescription for generalizing an equation concerning a matter field in SR to one in GR. It's supposed to guarantee the Poincaré invariance of equations arising from it on some spacetime region (Read et al, 2018, Appendix A), yet, as shown at the end of section 3.2 , this does not even entail that there are approximate local spacetime symmetries on that region. So, it can't be all that's needed to explain the success of SR matter theories, which requires showing that particular solutions to the dynamical equations - what experiments ultimately measure, after all—in some relevant sense 
approximate those of GR. ${ }^{16}$ Moreover, that relativistic spacetimes have approximate local Poincaré symmetries does not guarantee that matter fields in GR behave locally in the relevant respects just like matter fields in SR. A full explanation of the local success of SR from GR-not just of the geometry of Minkowski spacetime but of matter theories formulated on it-will require something more.

There seem to be at least two components needed. First one must find a less "ambiguous" procedure for identifying the correct GR generalizations of SR dynamical equations for matter fields. Minimal coupling is considered "ambiguous" because it yields syntactically and semantically inequivalent results when applied to semantically equivalent but syntactically distinct equations. To say that two equations are semantically equivalent is just to say that they share the same intension. Yet two such (non-identical) equations can differ regarding whether they are minimally coupled equations. Thus, more properly, the problem with minimal coupling, particularly in its application to reduction, is its hyperintensionality (Nolan, 2014): it draws distinctions between cointensional properties (Hoffmann-Kolss, 2015). What justification does such a prescription have? I am skeptical that there is a justification or a "non-ambiguous" procedure in this sense-cf. the analogous problem for understanding how to quantize a classical theory (Feintzeig, 2017) or to generalize a spacetime theory to different dimensions (Fletcher et al, 2018). Perhaps therefore the problem is better approached the other way around: identify matter equations in GR that have matter equations of SR as a special case in Minkowski spacetime, then select among those the ones that have in fact been successfully applied.

Once one provides these candidates, one can then develop the second component: a better account of what it means for matter fields in a region of one spacetime to approximate those in a region of another. Here the methods of Fletcher $(2014,2018 \mathrm{a})$ applied in section 3 to describe this for the spacetime metric and its derivatives ought to be of use. Although appeals to what one can detect with one's instruments here are surely correct, a more detailed explanation will surely be welcome.

Acknowledgements Thanks to Dennis Lehmkuhl, one of whose questions to me during the September, 2017 "Thinking About Space and Time" conference in Bern spurred the idea of this paper. Thanks also to James Read, Chris Wüthrich, and an anonymous referee for their comments, and especially to Jim Weatherall for suggesting a way to formalize a part of the proof of the main theorem and catching a serious error in a previous version thereof. Part of the research leading to this essay was written with the support from a Marie Curie Fellowship (PIIF-GA-2013-628533).

${ }^{16}$ Cf. the demand of Sonego and Faraoni (1993, p. 1185) for a real scalar field satisfying the homogeneous screened Poisson equation: "We require that the physical properties of wave propagation - rather than the form of the wave equation-should reduce locally to those valid in flat spacetime. More precisely, we require that the physical features of the solutions be locally the same in both cases." 


\section{References}

Brown HR (1997) On the role of special relativity in general relativity. International Studies in the Philosophy of Science 11(1):67-81

Brown HR (2005) Physical Relativity: Space-time structure from a dynamical perspective. Clarendon Press, Oxford

Brown HR, Pooley O (2001) The origin of the spacetime metric: Bell's 'Lorentzian pedagogy' and its significance in general relativity. In: Callender C, Huggett $N$ (eds) Physics meets philosophy at the Planck scale: Contemporary theories in quantum gravity, Cambridge University Press, Cambridge, pp 256-272

Carroll S, Lim E (2004) Lorentz-violating vector fields slow the universe down. Physical Review D 70:123,525

Ehlers J (1986) On limit relations between, and approximative explanations of, physical theories. In: Barcan Marcus R, Dorn GJW, Weingartner P (eds) Logic, Methodology and Philosophy of Science VII, Elsevier, Amsterdam, pp 387-403

Einstein A (1923) The foundation of the general theory of relativity. In: The Prinicple of Relativity, Methuen, pp 109-164, trans. Perrett W, Jeffery GB

Einstein A (1956) The Meaning of Relativity, 5th edn. Princeton University Press, Princeton, trans. Adams EP, Strauss EG, Bargmann S

Feintzeig BH (2017) On theory construction in physics: Continuity from classical to quantum. Erkenntnis 82(6):1195-1210

Fletcher SC (2014) Similarity and spacetime: Studies in intertheoretic reduction and physical significance. $\mathrm{PhD}$ thesis, University of California, Irvine

Fletcher SC (2016) Similarity, topology, and physical significance in relativity theory. The British Journal for the Philosophy of Science 67(2):365-389

Fletcher SC (2018a) Approximate symmetry of spacetime structure, unpublished manuscript

Fletcher SC (2018b) On representational capacities, with an application to general relativity. Foundations of Physics (forthcoming):1-22, URL https://doi.org/10.1007/s10701-018-0208-6

Fletcher SC, Manchak J, Schneider MD, Weatherall JO (2018) Would two dimensions be world enough for spacetime? Studies in History and Philosophy of Modern Physics 63:100-113

Hall GS (2004) Symmetry and Curvature Structure in General Relativity. World Scientific, Singapore

Hoffmann-Kolss V (2015) On a sufficient condition for hyperintensionality. Philosophical Quarterly 65(260):336-354

Jacobson T, Speranza AJ (2015) Variations on an aetherial theme. Physical Review D 92:044,030

Janssen M (2014) "No success like failure...": Einstein's quest for general relativity, 1907-1920. In: Janssen M, Lehner C (eds) The Cambridge Companion to Einstein, Cambridge University Press, Cambridge, pp 167-227

Malament DB (2007) Classical relativity theory. In: Butterfield J, Earman J (eds) Philosophy of Physics, Handbook of the Philosophy of Science, vol A, Elsevier, Amsterdam, pp 229-274

Nickles T (1973) Two concepts of intertheoretic reduction. The Journal of Philosophy 70(7):181-201

Nolan D (2014) Hyperintensional metaphysics. Philosophical Studies 171(1):149-160

Norton JD (1984) How Einstein found his field equations: 1912-1915. Historical Studies in the Physical Sciences 14(2):253-316

Norton JD (1993) General covariance and the foundations of general relativity: eight decades of dispute. Reports on Progress in Physics 56:791-858

Pauli W (1958) Theory of Relativity. Pergamon, trans. Field G

Read J, Brown HR, Lehmkuhl D (2018) Two miracles of general relativity. Studies in History and Philosophy of Modern Physics 64:14-25

Renn J, Sauer T (1998) Heuristics and mathematical representation in Einstein's search for a gravitational field equation. In: Goenner H, Renn J, Ritter J, Sauer T (eds) The Expanding Worlds of General Relativity, Einstein Studies, vol. 7, Birkhäuser, Boston, pp 87-125 
Rosaler J (2019) Reduction as an a posteriori relation. The British Journal for the Philosophy of Science 70(1):269-299

Schrödinger E (1950) Space-time Structure. Cambridge University Press, Cambridge

Sonego S, Faraoni V (1993) Coupling to the curvature for a scalar field from the equivalence principle. Classical and Quantum Gravity 10(6):1185-1187

Torretti R (1996) Relativity and Geometry, Dover edn. Dover, Mineola, NY

Torretti R (1999) The Philosophy of Physics. Cambridge University Press, Cambridge

Wald RM (1984) General Relativity. University of Chicago Press, Chicago 